\title{
HARDY UNCERTAINTY PRINCIPLE, CONVEXITY AND PARABOLIC EVOLUTIONS
}

\author{
L. ESCAURIAZA, C. E. KENIG, G. PONCE, AND L. VEGA
}

\begin{abstract}
We give a new proof of the $L^{2}$ version of Hardy's uncertainty principle based on calculus and on its dynamical version for the heat equation. The reasonings rely on new log-convexity properties and the derivation of optimal Gaussian decay bounds for solutions to the heat equation with Gaussian decay at a future time. We extend the result to heat equations with lower order variable coefficient.
\end{abstract}

\section{INTRODUCTION}

In this paper we continue the study in [18, 6, 8, 9, 10, 11, related to the Hardy uncertainty principle and its relation to unique continuation properties for some evolutions.

One of our motivations came from a well known result due to G. H. Hardy ([14, [21. pp. 131]), which concerns the decay of a function $f$ and its Fourier transform,

$$
\widehat{f}(\xi)=(2 \pi)^{-\frac{n}{2}} \int_{\mathbb{R}^{n}} e^{-i \xi \cdot x} f(x) d x .
$$

If $f(x)=O\left(e^{-|x|^{2} / \beta^{2}}\right), \widehat{f}(\xi)=O\left(e^{-4|\xi|^{2} / \alpha^{2}}\right)$ and $1 / \alpha \beta>1 / 4$, then $f \equiv 0$. Also, if $1 / \alpha \beta=1 / 4, f$ is a constant multiple of $e^{-|x|^{2} / \beta^{2}}$.

As far as we know, the known proofs for this result and its variants - before the one in [18, 6, 9, 10, 11] - use complex analysis (the Phragmén-Lindelöf principle). There has also been considerable interest in a better understanding of this result and on extensions of it to other settings: [3, [15, 20, 1] and 20.

The result can be rewritten in terms of the free solution of the Schrödinger equation

$$
i \partial_{t} u+\triangle u=0, \text { in } \mathbb{R}^{n} \times(0,+\infty),
$$

with initial data $f$,

$$
u(x, t)=(4 \pi i t)^{-\frac{n}{2}} \int_{\mathbb{R}^{n}} e^{\frac{i|x-y|^{2}}{4 t}} f(y) d y=(2 \pi i t)^{-\frac{n}{2}} e^{\frac{i|x|^{2}}{4 t}} e^{\frac{i|\cdot|^{2}}{4 t}} f\left(\frac{x}{2 t}\right),
$$

in the following way:

$$
\text { If } u(x, 0)=O\left(e^{-|x|^{2} / \beta^{2}}\right), u(x, T)=O\left(e^{-|x|^{2} / \alpha^{2}}\right) \text { and } T / \alpha \beta>1 / 4 \text {, then } u \equiv 0 \text {. }
$$
Also, if $T / \alpha \beta=1 / 4, u$ has as initial data a constant multiple of $e^{-\left(1 / \beta^{2}+i / 4 T\right)|y|^{2}}$.

1991 Mathematics Subject Classification. Primary: 35B05. Secondary: 35B60.

Key words and phrases. uncertainty principle, heat equation.

The first and fourth authors are supported by the grants MTM2014-53145-P and IT641-13 (GIC12/96), the second author by NSF grant DMS-0456583, and the fourth author is also supported by SEV-2013-0323. 
The corresponding results in terms of $L^{2}$-norms, established in [4, are the following:

$$
\begin{aligned}
& \text { If } e^{|x|^{2} / \beta^{2}} f, e^{4|\xi|^{2} / \alpha^{2}} \widehat{f} \text { are in } L^{2}\left(\mathbb{R}^{n}\right) \text { and } 1 / \alpha \beta \geq 1 / 4 \text {, then } f \equiv 0 \text {. } \\
& \text { If } e^{|x|^{2} / \beta^{2}} u(x, 0), e^{|x|^{2} / \alpha^{2}} u(x, T) \text { are in } L^{2}\left(\mathbb{R}^{n}\right) \text { and } T / \alpha \beta \geq 1 / 4 \text {, then } u \equiv 0 .
\end{aligned}
$$

In [10] we proved a uniqueness result in this direction for variable coefficients Schrödinger evolutions

$$
\partial_{t} u=i(\triangle u+V(x, t) u), \text { in } \mathbb{R}^{n} \times[0, T] .
$$

with bounded potentials $V$ verifying, $V(x, t)=V_{1}(x)+V_{2}(x, t)$, with $V_{1}$ real-valued and

or

$$
\sup _{[0, T]}\left\|e^{T^{2}|x|^{2} /(\alpha t+\beta(T-t))^{2}} V_{2}(t)\right\|_{L^{\infty}\left(\mathbb{R}^{n}\right)}<+\infty
$$

$$
\lim _{R \rightarrow+\infty} \int_{0}^{T}\|V(t)\|_{L^{\infty}\left(\mathbb{R}^{n} \backslash B_{R}\right)} d t=0 .
$$

More precisely, we showed that the only solution $u$ to (1.1) in $C\left([0, T], L^{2}\left(\mathbb{R}^{n}\right)\right)$, which verifies

$$
\left\|e^{|x|^{2} / \beta^{2}} u(0)\right\|_{L^{2}\left(\mathbb{R}^{n}\right)}+\left\|e^{|x|^{2} / \alpha^{2}} u(T)\right\|_{L^{2}\left(\mathbb{R}^{n}\right)}<+\infty
$$

is the zero solution, when $T / \alpha \beta>1 / 4$. When $T / \alpha \beta=1 / 4$, we found a complex valued potential potential $V$ with

$$
|V(x, t)| \lesssim \frac{1}{1+|x|^{2}}, \text { in } \mathbb{R}^{n} \times[0, T]
$$

and a nonzero smooth solution $u$ in $C^{\infty}\left([0, T], \mathcal{S}\left(\mathbb{R}^{n}\right)\right)$ of (1.1) with

$$
\left\|e^{|x|^{2} / \beta^{2}} u(0)\right\|_{L^{2}\left(\mathbb{R}^{n}\right)}+\left\|e^{|x|^{2} / \alpha^{2}} u(T)\right\|_{L^{2}\left(\mathbb{R}^{n}\right)}<+\infty .
$$

Thus, we established in 10 that the optimal version of Hardy's Uncertainty Principle in terms of $L^{2}$-norms holds for solutions to (1.1) holds when $T / \alpha \beta>1 / 4$ for many general bounded potentials, while it can fail for some complex-valued potentials in the end-point case, $T / \alpha \beta=1 / 4$. Finally, in [11 we showed that the reasonings in [18, 6, 8, 9, 10, 11] provide the first proof (up to the end-point case) that we know of Hardy's uncertainty principle for the Fourier transform without the use of holomorphic functions.

The Hardy uncertainty principle also has a dynamical version associated to the heat equation,

with initial data $f$,

$$
\partial_{t} u-\Delta u=0, \text { in } \mathbb{R}^{n} \times(0,+\infty),
$$

$u(x, t)=(4 \pi t)^{-n / 2} \int_{\mathbb{R}^{n}} e^{-|x-y|^{2} / 4 t} f(y) d y, \widehat{u}(\xi, t)=e^{-t|\xi|^{2}} \widehat{f}(\xi), x, \xi \in \mathbb{R}^{n}, t>0$.

In particular, its $L^{\infty}$ and $L^{2}$ versions yield the following statements:

If $u(0)$ is a finite measure in $\mathbb{R}^{n}, u(x, T)=O\left(e^{-|x|^{2} / \delta^{2}}\right)$ and $\delta<\sqrt{4 T}$, then $f \equiv 0$. Also, if $\delta=\sqrt{4 T}$, then $u(0)$ is a multiple of the Dirac delta function.

If $u(0)$ is in $L^{2}\left(\mathbb{R}^{n}\right),\left\|e^{|x|^{2} / \delta^{2}} u(T)\right\|_{L^{2}\left(\mathbb{R}^{n}\right)}$ is finite and $\delta \leq \sqrt{4 T}$, then $u \equiv 0$.

In [9, Theorem 4] we proved that a dynamical $L^{2}$-version of Hardy uncertainty principle holds for solutions $u$ in $C\left([0, T], L^{2}\left(\mathbb{R}^{n}\right)\right) \cap L^{2}\left([0, T], H^{1}\left(\mathbb{R}^{n}\right)\right)$ to

$$
\partial_{t} u=\Delta u+V(x, t) u, \text { in } \mathbb{R}^{n} \times[0, T],
$$


when $V$ is any bounded complex potential in $\mathbb{R}^{n} \times[0, T]$ and $\delta<\sqrt{T}$. Here, we find the optimal interior Gaussian decay over $[0,1]$ for solutions to (1.2) with

$$
\left\|e^{|x|^{2} / \delta^{2}} u(T)\right\|_{L^{2}\left(\mathbb{R}^{n}\right)}<+\infty,
$$

when $\delta>\sqrt{4 T}$ and derive from it the full dynamical $L^{2}$ version of the Hardy uncertainty principle for solutions to (1.2), reaching the end-point case, $\delta=\sqrt{4 T}$.

Theorem 1. Assume that $u$ in $C\left([0, T], L^{2}\left(\mathbb{R}^{n}\right)\right) \cap L^{2}\left([0, T], H^{1}\left(\mathbb{R}^{n}\right)\right)$ verifies (1.2) with $V$ in $L^{\infty}\left(\mathbb{R}^{n} \times[0, T]\right)$. Assume that

$$
\left\|e^{T|x|^{2} / 4\left(T^{2}+R^{2}\right)} u(T)\right\|_{L^{2}\left(\mathbb{R}^{n}\right)}<+\infty
$$

for some $R>0$. Then, there is a universal constant $N$ such that

$$
\begin{aligned}
& \text { (1.4) } \sup _{[0, T]}\left\|e^{t|x|^{2} / 4\left(t^{2}+R^{2}\right)} u(t)\right\|_{L^{2}\left(\mathbb{R}^{n}\right)} \\
& \leq e^{N\left(1+T^{2}\|V\|_{L^{\infty}\left(\mathbb{R}^{n} \times[0, T]\right)}^{2}\right)}\left[\|u(0)\|_{L^{2}\left(\mathbb{R}^{n}\right)}+\left\|e^{T|x|^{2} / 4\left(T^{2}+R^{2}\right)} u(T)\right\|_{L^{2}\left(\mathbb{R}^{n}\right)}\right] . \\
& \text { Moreover, } u \text { must be identically zero when }\left\|e^{|x|^{2} / 4 T} u(T)\right\|_{L^{2}\left(\mathbb{R}^{n}\right)} \text { is finite. }
\end{aligned}
$$

Theorem 1 is optimal because

$$
u_{R}(x, t)=(t-i R)^{-\frac{n}{2}} e^{-|x|^{2} / 4(t-i R)}=(t-i R)^{-\frac{n}{2}} e^{-(t+i R)|x|^{2} / 4\left(t^{2}+R^{2}\right)},
$$

is a solution to the heat equation and for each fixed $t>0, t / 4\left(t^{2}+R^{2}\right)$ is decreasing in the $R$-variable for $R>0$. Also, observe that $t / 4\left(t^{2}+R^{2}\right)$ attains its maximum value in the interior of $[0, T]$, when $R \neq T$,

Notice that the finiteness condition on condition on $\left\|e^{|x|^{2} / 4 T} u(T)\right\|_{L^{2}\left(\mathbb{R}^{n}\right)}$ is independent of the size of the potential or the dimension and that we do not assume any regularity or strong decay of the potentials.

This improvement of our results in [9, Theorem 4] on the relation between Hardy uncertainty principle and its dynamical version for parabolic evolutions comes from a better understanding of the solutions to (1.2) which have Gaussian decay and of the adaptation to the parabolic context of the same kind of log-convexity arguments that we used in [10, to derive the dynamical version of the Hardy uncertainty principle for Schrödinger evolutions.

We have not tried to extend the results in Theorems 1 to parabolic evolutions with nonzero drift terms

$$
\partial_{t} u=\Delta u+W(x, t) \cdot \nabla u+V(x, t) u .
$$

We expect that similar methods will yield analogue results for solutions to (1.6) (See [5] for initial results following the approach initiated in [18] and [6] for the case of Schr̈odiger evolutions).

In what follows, $N$ denotes a universal constant depending at most on the dimension, $N_{a, \xi, \ldots}$ a constant depending on the parameters $a, \xi, \ldots$ In section 2 we give three Lemmas which are necessary for our proof in section 3 of Theorem 1 .

\section{A feW Lemmas}

In the sequel

$$
(f, g)=\int_{\mathbb{R}^{n}} f \bar{g} d x,\|f\|^{2}=(f, f) \text { and }\|V\|_{\infty}=\|V\|_{L^{\infty}\left(\mathbb{R}^{n} \times[0,1]\right)} .
$$


In Lemma 1, $\mathcal{S}$ and $\mathcal{A}$ denote respectively a symmetric and skew-symmetric bounded linear operators on $\mathcal{S}\left(\mathbb{R}^{n}\right)$. Both are allowed to depend smoothly on the time-variable, $\mathcal{S}_{t}=\partial_{t} \mathcal{S}$ and $[\mathcal{S}, \mathcal{A}]$ is the space commutator of $\mathcal{S}$ and $\mathcal{A}$. The reader can find a proof of Lemma 1] in [10, Lemma 2].

Lemma 1. Let $\mathcal{S}$ and $\mathcal{A}$ be as above, $f$ lie in $C^{\infty}\left([c, d], \mathcal{S}\left(\mathbb{R}^{n}\right)\right)$ and $\gamma:[c, d] \longrightarrow$ $(0,+\infty)$ be a smooth function such that

$$
\left(\gamma \mathcal{S}_{t} f(t)+\gamma[\mathcal{S}, \mathcal{A}] f(t)+\dot{\gamma} \mathcal{S} f(t), f(t)\right) \geq 0, \text { when } c \leq t \leq d .
$$

Then, if $H(t)=\|f(t)\|^{2}$ and $\epsilon>0$

$$
H(t)+\epsilon \leq(H(c)+\epsilon)^{\theta(t)}(H(d)+\epsilon)^{1-\theta(t)} e^{M_{\epsilon}(t)+2 N_{\epsilon}(t)} \text {, when } c \leq t \leq d,
$$

where $M_{\epsilon}$ verifies

$$
\begin{gathered}
\partial_{t}\left(\gamma \partial_{t} M_{\epsilon}\right)=-\gamma \frac{\left\|\partial_{t} f-\mathcal{S} f-\mathcal{A} f\right\|^{2}}{H+\epsilon}, \text { in }[c, d], \quad M_{\epsilon}(c)=M_{\epsilon}(d)=0, \\
N_{\epsilon}=\int_{c}^{d}\left|R e \frac{\left(\partial_{s} f(s)-\mathcal{S} f(s)-\mathcal{A} f(s), f(s)\right)}{H(s)+\epsilon}\right| d s
\end{gathered}
$$

and

$$
\theta(t)=\frac{\int_{t}^{d} \frac{d s}{\gamma}}{\int_{c}^{d} \frac{d s}{\gamma}} .
$$

A calculation (see formulae (2.12), (2.13) and (2.14) in [9] with $\gamma=1$ ) shows that given smooth functions $a:[0,1] \longrightarrow[0,+\infty), b:[0,1] \longrightarrow \mathbb{R}$ and $T:[0,1] \longrightarrow \mathbb{R}$, and $\xi$ in $\mathbb{R}^{n}$

$$
e^{a(t)|x|^{2}+b(t) x \cdot \xi-T(t)|\xi|^{2}}\left(\partial_{t}-\triangle\right) e^{-a(t)|x|^{2}-b(t) x \cdot \xi+T(t)|\xi|^{2}}=\partial_{t}-\mathcal{S}-\mathcal{A},
$$

where $\mathcal{S}$ and $\mathcal{A}$ are the symmetric and skew-symmetric linear bounded operators on $\mathcal{S}\left(\mathbb{R}^{n}\right)$ given by

$$
\begin{aligned}
& \mathcal{S}=\Delta+\left(a^{\prime}+4 a^{2}\right)|x|^{2}+\left(b^{\prime}+4 a b\right) x \cdot \xi+\left(b^{2}-T^{\prime}\right)|\xi|^{2}, \\
& \mathcal{A}=-2(2 a x+b \xi) \cdot \nabla-2 n a .
\end{aligned}
$$

and

$$
\begin{aligned}
\mathcal{S}_{t}+[\mathcal{S}, \mathcal{A}]= & -8 a \Delta+\left(a^{\prime \prime}+16 a a^{\prime}+32 a^{3}\right)|x|^{2} \\
& +\left(b^{\prime \prime}+8 a b^{\prime}+8 a^{\prime} b+32 a^{2} b\right) x \cdot \xi+\left(8 a b^{2}+4 b b^{\prime}-T^{\prime \prime}\right)|\xi|^{2} .
\end{aligned}
$$

In Lemma 2 we make choices of $a, b$ and $T$ which make non-negative the selfadjoint operator

$$
e^{8 A}\left(\mathcal{S}_{t}+[\mathcal{S}, \mathcal{A}]\right)+\left(e^{8 A}\right)^{\prime} \mathcal{S}
$$

where $A$ denotes an anti-derivative of $a$ in $[0,1]$ with $A(1)=0$, .

Lemma 2. Let $a:[0,1] \longrightarrow \mathbb{R}$ be a smooth function verifying

$$
\left(e^{8 A} a\right)^{\prime \prime} \geq 0, \text { in }[0,1],
$$

and let $b$ and $T$ be the solutions to

$$
\left\{\begin{array}{l}
\left(e^{8 A} b\right)^{\prime \prime}=2\left(e^{8 A} a\right)^{\prime \prime}, \text { in }[0,1] \\
b(0)=b(1)=0
\end{array}\right.
$$


and

$$
\left\{\begin{array}{l}
\left(e^{8 A} T^{\prime}\right)^{\prime}=2\left(e^{8 A} b^{2}\right)^{\prime}-\left(e^{8 A} a\right)^{\prime \prime}, \text { in }[0,1], \\
T(0)=T(1)=0 .
\end{array}\right.
$$

Then,

$$
\left(e^{8 A} \mathcal{S}_{t} f+e^{8 A}[\mathcal{S}, \mathcal{A}] f+\left(e^{8 A}\right)^{\prime} \mathcal{S} f, f\right) \geq 0, \text { when } f \in \mathcal{S}\left(\mathbb{R}^{n}\right) \text { and } 0 \leq t \leq 1 .
$$

Proof. From (2.1), (2.3), the identities

$$
\begin{aligned}
& \left(e^{8 A} a\right)^{\prime \prime}=e^{8 A}\left(a^{\prime \prime}+24 a a^{\prime}+64 a^{3}\right) . \\
& \left(e^{8 A} b\right)^{\prime \prime}=e^{8 A}\left(b^{\prime \prime}+16 a b^{\prime}+8 a^{\prime} b+64 a^{2} b\right) . \\
& \left(e^{8 A} b^{2}\right)^{\prime}=e^{8 A}\left(8 a b^{2}+2 b b^{\prime}\right),
\end{aligned}
$$

and the definitions of $b$ and $T$, we have

$$
\begin{gathered}
e^{8 A}\left(\mathcal{S}_{t}+[\mathcal{S}, \mathcal{A}]\right)+\left(e^{8 A}\right)^{\prime} \mathcal{S} \\
=\left(e^{8 A} a\right)^{\prime \prime}|x|^{2}+\left(e^{8 A} b\right)^{\prime \prime} x \cdot \xi+\left(2\left(e^{8 A} b^{2}\right)^{\prime}-\left(e^{8 A} T^{\prime}\right)^{\prime}\right)|\xi|^{2} \\
=\left(e^{8 A} a\right)^{\prime \prime}\left(|x|^{2}+2 x \cdot \xi+|\xi|^{2}\right)=\left(e^{8 A} a\right)^{\prime \prime}|x+\xi|^{2} .
\end{gathered}
$$

The later and (2.4) implies Lemma 2 ,

In the next Lemma we assume that $u$ in $C\left([0,1], L^{2}\left(\mathbb{R}^{n}\right)\right) \cap L^{2}\left([0,1], H^{1}\left(\mathbb{R}^{n}\right)\right)$ verifies (1.2) in $\mathbb{R}^{n} \times(0,1]$ and

$$
\left\|e^{|x|^{2} / \delta^{2}} u(1)\right\|<+\infty .
$$

Lemma 3. Let $a:[0,1] \longrightarrow[0,+\infty)$ be a smooth function with $a(0)=0, a(1)=$ $1 / \delta^{2},\left(e^{8 A} a\right)^{\prime \prime}>0$ in $[0,1]$ and

$$
\sup _{[0,1]}\left\|e^{(a(t)-\epsilon)|x|^{2}} u(t)\right\|<+\infty \text {, when } 0<\epsilon \leq 1 .
$$

Then, there is a universal constant $N$ such that for $b$ and $T$ as in (2.5) and (2.6),

$$
\left\|e^{a(t)|x|^{2}+b(t) x \cdot \xi-T(t)|\xi|^{2}} u(t)\right\| \leq e^{N\left(1+\|V\|_{\infty}^{2}\right)}\left(\|u(0)\|+\left\|e^{|x|^{2} / \delta^{2}} u(1)\right\|\right),
$$

when $\xi$ is in $\mathbb{R}^{n}$ and $0 \leq t \leq 1$.

Proof. For $\xi$ in $\mathbb{R}^{n}$ and $\epsilon>0$, set

$$
f_{\epsilon}(x, t)=e^{a_{\epsilon}|x|^{2}+b_{\epsilon} x \cdot \xi-T_{\epsilon}|\xi|^{2}} u(x, t),
$$

with $a_{\epsilon}=a-\epsilon, A_{\epsilon}=A+\epsilon(1-t)$, and with $b_{\epsilon}$ and $T_{\epsilon}$ as in Lemma 2 but with $a$ and $A$ replaced by $a_{\epsilon}$ and $A_{\epsilon}$ respectively. The local Schauder estimates for solutions to (1.2) show that

$$
\begin{aligned}
& r|\nabla u(x, t)|+r^{2}\left(f_{B_{r}(x) \times\left(t-r^{2}, t\right]}\left|\partial_{s} u\right|^{p}+\left|D^{2} u\right|^{p} d y d s\right)^{\frac{1}{p}} \\
& \quad \leq N_{p}\left(1+r^{2}\|V\|_{L^{\infty}\left(\mathbb{R}^{n} \times[0,1]\right)}\right) f_{B_{2 r}(x) \times\left(t-4 r^{2}, t\right]}|u| d y d s
\end{aligned}
$$


for $1<p<\infty, 0<r \leq \sqrt{t} / 2,0<t \leq 1$. Thus, $f_{\epsilon}$ is in $W_{2}^{2,1}\left(\mathbb{R}^{n} \times[\varrho, 1]\right)$ and verifies

$$
\begin{aligned}
& \sup _{[0,1]}\left\|f_{\epsilon}(t)\right\| \leq N_{a, \epsilon, \xi} \sup _{[0,1]}\left\|e^{\left(a-\frac{\epsilon}{2}\right)|x|^{2}} u(t)\right\|, \\
& \sup _{[\varrho, 1]}\left\|\nabla f_{\epsilon}(t)\right\| \leq N_{a, \epsilon, \xi, \varrho} \sup _{[0,1]}\left\|e^{\left(a-\frac{\epsilon}{2}\right)|x|^{2}} u(t)\right\|
\end{aligned}
$$

for $0<\varrho \leq \frac{1}{2}$ and

$$
\partial_{t} f_{\epsilon}-\mathcal{S}_{\epsilon} f_{\epsilon}-\mathcal{A}_{\epsilon} f_{\epsilon}=V(x, t) f_{\epsilon}, \text { in } \mathbb{R}^{n} \times(0,1],
$$

where $\mathcal{S}_{\epsilon}$ and $\mathcal{A}_{\epsilon}$ are the operators defined in (2.1) and (2.2) with $a, A, b$ and $T$ replaced by $a_{\epsilon}, A_{\epsilon}, b_{\epsilon}$ and $T_{\epsilon}$ respectively. Also, (2.7), the equation (2.8) verified by $f_{\epsilon}$ and [22, Lemma 1.2] show that $f_{\epsilon}$ is in $C\left((0,1], L^{2}\left(\mathbb{R}^{n}\right)\right)$.

Extend $f_{\epsilon}$ as zero outside $\mathbb{R}^{n} \times[0,1]$ and let $\theta$ in $C^{\infty}\left(\mathbb{R}^{n+1}\right)$ be a mollifier supported in the unit ball of $\mathbb{R}^{n+1}$. For $0<\rho \leq \frac{1}{4}$, set $f_{\epsilon, \rho}=f_{\epsilon} * \theta_{\rho}$ and

$$
\theta_{\rho}^{x, t}(y, s)=\rho^{-n-1} \theta\left(\frac{x-y}{\rho}, \frac{t-s}{\rho}\right) .
$$

Then, $f_{\epsilon, \rho}$ is in $C^{\infty}\left([0,1], \mathcal{S}\left(\mathbb{R}^{n}\right)\right)$ and for $x$ in $\mathbb{R}^{n}$ and $\rho \leq t \leq 1-\rho$,

$$
\begin{aligned}
& \left(\partial_{t} f_{\epsilon, \rho}-\mathcal{S}_{\epsilon} f_{\epsilon, \rho}-\mathcal{A}_{\epsilon} f_{\epsilon, \rho}\right)(x, t)=\left(V f_{\epsilon}\right) * \theta_{\rho}(x, t) \\
& +\int f_{\epsilon}\left(q_{\epsilon}(y, s, \xi)-q_{\epsilon}(x, t, \xi)\right) \theta_{\rho}^{x, t} d y d s \\
& +\int \nabla_{y} f_{\epsilon} \cdot\left[\left(a_{\epsilon}(t) x+2 b_{\epsilon}(t) \xi\right)-\left(a_{\epsilon}(s) y+2 b_{\epsilon}(s) \xi\right)\right] \theta_{\rho}^{x, t} d y d s,
\end{aligned}
$$

with

$$
\begin{aligned}
q_{\epsilon}(x, t, \xi)=\left(a_{\epsilon}^{\prime}(t)+\right. & \left.4 a_{\epsilon}^{2}(t)\right)|x|^{2} \\
& +\left(b_{\epsilon}^{\prime}(t)+4 a_{\epsilon}(t) b_{\epsilon}(t)\right) x \cdot \xi+\left(b_{\epsilon}^{2}(t)-T_{\epsilon}^{\prime}(t)\right)|\xi|^{2}-2 n a_{\epsilon}(t) .
\end{aligned}
$$

The last identity gives,

$$
\left(\partial_{t} f_{\epsilon, \rho}-\mathcal{S}_{\epsilon} f_{\epsilon, \rho}-\mathcal{A}_{\epsilon} f_{\epsilon, \rho}\right)(x, t)=\left(V f_{\epsilon}\right) * \theta_{\rho}(x, t)+A_{\epsilon, \rho}(x, t),
$$

in $\mathbb{R}^{n} \times[\rho, 1-\rho]$, where $A_{\epsilon, \rho}$ denotes the sum of the second and third integrals in the right hand side of (2.9) . Moreover, from (2.7) there is $N_{a, \epsilon, \xi, \varrho}$ such that for $0<\varrho<\frac{1}{2}$ and $0<\rho \leq \varrho$,

$$
\sup _{[\varrho, 1-\varrho]}\left\|A_{\epsilon, \rho}(t)\right\|_{L^{2}\left(\mathbb{R}^{n}\right)} \leq \rho N_{a, \epsilon, \xi, \varrho} \sup _{[-1,1]}\left\|e^{\left(a(t)-\frac{\epsilon}{2}\right)|x|^{2}} u(t)\right\| .
$$

Also, $\left(e^{8 A_{\epsilon}} a_{\epsilon}\right)^{\prime \prime}>0$ in $[0,1]$, when $0<\epsilon \leq \epsilon_{a}$, and from Lemma 2 we can apply to $\mathcal{S}_{\epsilon}, \mathcal{A}_{\epsilon}$ and $f_{\epsilon, \rho}$, the conclusions of Lemma 1 with $[c, d]=[\varrho, 1-\varrho], \gamma=e^{8 A_{\epsilon}}$ and $H_{\epsilon, \rho}(t)=\left\|f_{\epsilon, \rho}(t)\right\|^{2}$. Thus,

$$
H_{\epsilon, \rho}(t) \leq\left(H_{\epsilon, \rho}(\varrho)+H_{\epsilon, \rho}(1-\varrho)+2 \epsilon\right) e^{M_{\epsilon, \rho}(t)+2 N_{\epsilon, \rho}}, \text { when } \varrho \leq t \leq 1-\varrho,
$$

where $M_{\epsilon, \rho}$ verifies

$$
\left\{\begin{array}{l}
\partial_{t}\left(e^{8 A_{\epsilon}} \partial_{t} M_{\epsilon, \rho}\right)=-e^{8 A_{\epsilon}} \frac{\left\|\partial_{t} f_{\epsilon, \rho}-\mathcal{S}_{\epsilon} f_{\epsilon, \rho}-\mathcal{A}_{\epsilon} f_{\epsilon, \rho}\right\|^{2}}{H_{\epsilon, \rho}+\epsilon}, \text { in }[\varrho, 1-\varrho] \\
M_{\epsilon, \rho}(\varrho)=M_{\epsilon, \rho}(1-\varrho)=0
\end{array}\right.
$$

and

$$
N_{\epsilon, \rho}=\int_{\varrho}^{1-\varrho} \frac{\left\|\partial_{s} f_{\epsilon, \rho}(s)-\mathcal{S}_{\epsilon} f_{\epsilon, \rho}(s)-\mathcal{A}_{\epsilon} f_{\epsilon, \rho}(s)\right\|}{\sqrt{H_{\epsilon, \rho}(s)+\epsilon}} d s .
$$


HARDY UNCERTAINTY PRINCIPLE, CONVEXITY AND PARABOLIC EVOLUTIONS 7

We can now pass to the limit in (2.10), when $\rho$ tends to zero and derive that for $H_{\epsilon}(t)=\left\|f_{\epsilon}(t)\right\|^{2}, 0<\varrho \leq \frac{1}{2}$ and $0<\epsilon \leq \epsilon_{a}$, we have

$$
H_{\epsilon}(t) \leq\left[H_{\epsilon}(\varrho)+H_{\epsilon}(1-\varrho)+2 \epsilon\right] e^{M_{\epsilon}(t)+2\|V\|_{\infty}}, \text { in }[\varrho, 1-\varrho],
$$

with

$$
\left\{\begin{array}{l}
\partial_{t}\left(e^{8 A_{\epsilon}} \partial_{t} M_{\epsilon}\right)=-e^{8 A_{\epsilon} \frac{\left\|\partial_{t} f_{\epsilon}-\mathcal{S}_{\epsilon} f_{\epsilon}-\mathcal{A}_{\epsilon} f_{\epsilon}\right\|^{2}}{H_{\epsilon}}}, \text { in }[0,1] . \\
M_{\epsilon}(0)=M_{\epsilon}(1)=0,
\end{array}\right.
$$

By writing an explicit formula for the solution to (2.12), it follows from the monotonicity of $A$; i.e. $A^{\prime} \geq 0$ in $[0,1]$ and (2.8) that

$$
M_{\epsilon}(t) \leq N\left(1+\|V\|_{\infty}^{2}\right) \text {. }
$$

Also, there is $N_{a}>0$ such that $\left|b_{\epsilon}^{\prime}\right|+\left|T_{\epsilon}^{\prime}\right| \leq N_{a}$, when $0<\epsilon \leq \epsilon_{a}$. The later, the continuity of $f_{\epsilon}$ in $C\left((0,1], L^{2}\left(\mathbb{R}^{n}\right)\right)$ and the fact that $a(0)=b_{\epsilon}(0)=T_{\epsilon}(0)=0$ show, that for each fixed $\xi \in \mathbb{R}^{n}$ and all $0<\epsilon<\epsilon_{a}$, there is $\varrho_{\epsilon}$ with $\lim _{\epsilon \rightarrow 0^{+}} \varrho_{\epsilon}=0$ such that $H_{\epsilon}\left(1-\varrho_{\epsilon}\right) \leq H_{\epsilon}(1)+\epsilon$ and $H_{\epsilon}\left(\varrho_{\epsilon}\right) \leq \sup _{[0,1]}\|u(t)\|$. Thus, after taking $\varrho=\varrho_{\epsilon}$ in (2.11), we get

$$
\left\|e^{a_{\epsilon}(t)|x|^{2}+b_{\epsilon}(t) x \cdot \xi-T_{\epsilon}(t)|\xi|^{2}} u(t)\right\| \leq e^{N\left(1+\|V\|_{\infty}^{2}\right)}\left[\sup _{[0,1]}\|u(t)\|+\left\|e^{|x|^{2} / \delta^{2}} u(1)\right\|+3 \epsilon\right],
$$

for $\varrho_{\epsilon} \leq t \leq 1-\varrho_{\epsilon}$. Then, let $\epsilon \rightarrow 0^{+}$and recall the $L^{2}$ energy inequality verified by solutions to (1.2).

\section{Proof of Theorem 1}

Proof. By scaling it suffices to prove Theorem 1 when $T=1$. Assume first that $u$ in $C\left([0,1], L^{2}\left(\mathbb{R}^{n}\right)\right) \cap L^{2}\left([0,1], H^{1}\left(\mathbb{R}^{n}\right)\right)$ verifies (1.2) in $\mathbb{R}^{n} \times(0,1]$ and

$$
\left\|e^{|x|^{2} / \delta^{2}} u(1)\right\|<+\infty
$$

for some $\delta>2$. Following [9, Theorem 4], for $\alpha=1$ and $\beta=1+\frac{2}{\delta}$, define

$$
\widetilde{u}(x, t)=\left(\frac{\sqrt{\alpha \beta}}{\alpha(1-t)+\beta t}\right)^{\frac{n}{2}} u\left(\frac{\sqrt{\alpha \beta} x}{\alpha(1-t)+\beta t}, \frac{\beta t}{\alpha(1-t)+\beta t}\right) e^{\frac{(\alpha-\beta)|x|^{2}}{4(\alpha(1-t)+\beta t)}} .
$$

Then, $\widetilde{u}$ is in $C\left([0,1], L^{2}\left(\mathbb{R}^{n}\right)\right) \cap L^{2}\left([0,1], H^{1}\left(\mathbb{R}^{n}\right)\right)$ and from [9, Lemma 5] with $A+i B=1$

$$
\partial_{t} \widetilde{u}=\Delta \widetilde{u}+\widetilde{V}(x, t) \widetilde{u}, \text { in } \mathbb{R}^{n} \times(0,1]
$$

with

$$
\widetilde{V}(x, t)=\frac{\alpha \beta}{(\alpha(1-t)+\beta t)^{2}} V\left(\frac{\sqrt{\alpha \beta} x}{\alpha(1-t)+\beta t}, \frac{\beta t}{\alpha(1-t)+\beta t}\right) .
$$

Also, for $\gamma=\frac{1}{2 \delta}$

$$
\left\|e^{\gamma|x|^{2}} \widetilde{u}(0)\right\|=\|u(0)\| \text { and }\left\|e^{\gamma|x|^{2}} \widetilde{u}(1)\right\|=\left\|e^{|x|^{2} / \delta^{2}} u(1)\right\| .
$$

From the log-convexity property of $\left\|e^{\gamma|x|^{2}} \widetilde{u}(t)\right\|$ established in [9, Lemma 3], we know that

$$
\sup _{[0,1]}\left\|e^{\gamma|x|^{2}} \widetilde{u}(t)\right\| \leq e^{N\left(1+\|\widetilde{V}\|_{L^{\infty}\left(\mathbb{R}^{n} \times[0,1]\right)}^{2}\right)}\left(\left\|e^{\gamma|x|^{2}} \widetilde{u}(0)\right\|+\left\|e^{\gamma|x|^{2}} \widetilde{u}(1)\right\|\right) .
$$

The last claim in [9, Lemma 5] shows that with $s=\frac{\beta t}{\alpha(1-t)+\beta t}$,

$$
\left\|e^{\gamma|x|^{2}} \widetilde{u}(t)\right\|=\left\|e^{\left[\frac{\gamma \alpha \beta}{(\alpha s+\beta(1-s))^{2}}+\frac{\alpha-\beta}{4(\alpha s+\beta(1-s))}\right]|y|^{2}} u(s)\right\|, \text { for } 0 \leq t \leq 1 .
$$


From (3.1) and (3.2), we find that

$$
\sup _{[0,1]}\left\|e^{\frac{t|x|^{2}}{(\delta+2-2 t)^{2}}} u(t)\right\| \leq e^{N\left(1+\|V\|_{\infty}^{2}\right)}\left[\|u(0)\|+\left\|e^{|x|^{2} / \delta^{2}} u(1)\right\|\right] .
$$

We then begin an inductive procedure where at the $k$ th step we have constructed $k$ smooth functions, $a_{j}:[0,1] \longrightarrow[0,+\infty)$ verifying

$$
\begin{gathered}
0<a_{1}<a_{2}<\cdots<a_{k}<\cdots \leq \frac{1}{\delta^{2}-4}, \text { in }(0,1), \\
a_{j}(0)=0, a_{j}(1)=1 / \delta^{2},\left(e^{8 A_{j}} a_{j}\right)^{\prime \prime}>0, \text { in }[0,1], \\
\sup _{[0,1]}\left\|e^{a_{j}(t)|x|^{2}} u(t)\right\| \leq e^{N\left(1+\|V\|_{\infty}^{2}\right)}\left[\|u(0)\|+\left\|e^{|x|^{2} / \delta^{2}} u(1)\right\|\right],
\end{gathered}
$$

when $j=1, \ldots, k$, with $A_{j}^{\prime}=a_{j}, A_{j}(1)=0$. The case $k=1$ follows from (3.3) with $a_{1}(t)=t /(\delta+2-2 t)^{2}$. Assume now that $a_{1}, \ldots, a_{k}$ have been constructed and let $b_{k}$ and $T_{k}$ be the functions defined in Lemma 3 for $a=a_{k}$. Then,

$$
\begin{aligned}
& \left\|e^{a_{k}(t)|x|^{2}+b_{k}(t) x \cdot \xi-T_{k}(t)|\xi|^{2}} u(t)\right\|^{2} \\
& \quad \leq e^{2 N\left(1+\|V\|_{\infty}^{2}\right)}\left(\|u(0)\|+\left\|e^{|x|^{2} / \delta^{2}} u(1)\right\|\right)^{2},
\end{aligned}
$$

for $0 \leq t \leq 1$ and all $\xi \in \mathbb{R}^{n}$. Observe that (3.7) and the existence of the solutions $u_{R}$ defined in (1.5) imply that $T_{k}>0$ in $(0,1)$, when $\delta>2$. Otherwise, (3.7) implies that $u_{R} \equiv 0$, when $2 \sqrt{1+R^{2}}<\delta$.

For $\epsilon>0$, multiply (3.7) by $e^{-2 \epsilon T_{k}(t)|\xi|^{2}}$ and integrate the new inequality with respect to $\xi$ in $\mathbb{R}^{n}$. It gives,

$$
\sup _{[0,1]}\left\|e^{a_{k+1}^{\epsilon}(t)|x|^{2}} u(t)\right\| \leq\left(1+\frac{1}{\epsilon}\right)^{\frac{n}{4}} e^{N\left(1+\|V\|_{\infty}^{2}\right)}\left(\|u(0)\|+\left\|e^{|x|^{2} / \delta^{2}} u(1)\right\|\right),
$$

with

$$
a_{k+1}^{\epsilon}=a_{k}+\frac{b_{k}^{2}}{4(1+\epsilon) T_{k}} .
$$

On the other hand, $e^{8 A_{k}} b_{k}$ is strictly convex and $b_{k}<0$ in $[0,1]$,

$$
b_{k}(t)=2\left(a_{k}(t)-t e^{-8 A_{k}(t)} \delta^{-2}\right)
$$

and

$$
T_{k}(t)=2 \int_{0}^{t} b_{k}^{2}(s) d s-a_{k}(t)-8 \int_{0}^{t} a_{k}^{2}(s) d s-\alpha_{k} \int_{0}^{t} e^{-8 A_{k}(s)} d s
$$

with

$$
\alpha_{k}=\left(2 \int_{0}^{1} b_{k}^{2}(s) d s-\frac{1}{\delta^{2}}-8 \int_{0}^{1} a_{k}^{2}(s) d s\right)\left(\int_{0}^{1} e^{-8 A_{k}(s)} d s\right)^{-1} .
$$

The last two formulae and (3.4) show that there is $N_{\delta} \geq 1$, independent of $k \geq 1$, such that

$$
T_{k}(t) \leq 2\left(\int_{0}^{t} b_{k}^{2}(s) d s+N_{\delta}\right) \text { and } N_{\delta}+\frac{b_{k}}{2} \geq 1 \text {, in }[0,1] .
$$


Also, $\left(\left(a_{k}^{\prime}+4 a_{k}^{2}\right) e^{16 A_{k}}\right)^{\prime}=e^{8 A_{k}}\left(e^{8 A_{k}} a_{k}\right)^{\prime \prime},\left(a_{k}^{\prime}+4 a_{k}^{2}\right) e^{16 A_{k}}$ is non decreasing in $[0,1]$ and

$$
a_{k}^{\prime}+4 a_{k}^{2} \geq 0 \text { in }[0,1]
$$

Set then,

$$
a_{k+1}(t)=a_{k}(t)+\frac{b_{k}^{2}(t)}{8\left(\int_{0}^{t} b_{k}^{2}(s) d s+N_{\delta}\right)} .
$$

We have, $a_{k}<a_{k+1}$ in $(0,1), a_{k+1}(0)=0, a_{k+1}(1)=\frac{1}{\delta^{2}}$,

$$
A_{k+1}=A_{k}+\frac{1}{8} \log \left(\int_{0}^{t} b_{k}^{2}(s) d s+N_{\delta}\right)-\frac{1}{8} \log \left(\int_{0}^{1} b_{k}^{2}(s) d s+N_{\delta}\right),
$$

and

$$
\sup _{[0,1]}\left\|e^{\left(a_{k+1}(t)-\epsilon\right)|x|^{2}} u(t)\right\|<+\infty, \text { for all } \epsilon>0 .
$$

The identity $\left(e^{8 A}\right)^{\prime \prime \prime}=8\left(e^{8 A} a\right)^{\prime \prime}$ and (3.12) show that $\left(e^{8 A_{k+1}} a_{k+1}\right)^{\prime \prime}$ is a positive multiple of

$$
\begin{aligned}
\left(e^{8 A_{k}}\left(\int_{0}^{t} b_{k}^{2}(s) d s+N_{\delta}\right)\right)^{\prime \prime \prime} & =\left(e^{8 A_{k}}\right)^{\prime \prime \prime}\left(\int_{0}^{t} b_{k}^{2}(s) d s+N_{\delta}\right) \\
& +3\left(e^{8 A_{k}}\right)^{\prime \prime} b_{k}^{2}+6\left(e^{8 A_{k}}\right)^{\prime} b_{k} b_{k}^{\prime}+2 e^{8 A_{k}}\left(b_{k}^{\prime \prime} b_{k}+b_{k}^{\prime 2}\right)
\end{aligned}
$$

The equation verified by $b_{k}$ shows that the last sum is equal to

$$
\begin{aligned}
\left(e^{8 A_{k}}\right)^{\prime \prime \prime}\left(\int_{0}^{t} b_{k}^{2}(s) d s+N_{\delta}\right. & \left.+\frac{b_{k}}{2}\right) \\
& +8\left(a_{k}^{\prime}+8 a_{k}^{2}\right) e^{8 A_{k}} b_{k}^{2}+2 e^{8 A_{k}} b_{k}^{\prime 2}+16 e^{8 A_{k}} a_{k} b_{k} b_{k}^{\prime} .
\end{aligned}
$$

From (3.9) and (3.10), the above sum is bounded from below by

$$
\left(e^{8 A_{k}}\right)^{\prime \prime \prime}+2 e^{8 A_{k}}\left(4 a_{k} b_{k}+b_{k}^{\prime}\right)^{2}>0, \text { in }[0,1] .
$$

The later and Lemma 3 show that (3.6) holds up to $j=k+1$. Finally, because (3.10) holds with $k$ replaced by $k+1$,

$$
-\left(\frac{1}{a_{k+1}}\right)^{\prime}+4 \geq 0, \text { in }(0,1],
$$

and the integration of this identity over $[t, 1]$ shows that $a_{k+1}(t) \leq \frac{1}{\delta^{2}-4}$ in $(0,1)$.

Thus, there exists $a(t)=\lim _{k \rightarrow+\infty} a_{k}(t)$ and from (3.11), $\lim _{k \rightarrow+\infty} b_{k}(t)=0$. This and (3.8) show that

$$
a e^{8 A}=t \delta^{-2}, \text { in }[0,1] .
$$

Write $a(1)=1 / \delta^{2}$ as $1 / 4\left(1+R^{2}\right)$, for some $R>0$. Then, $a(t)=t / 4\left(t^{2}+R^{2}\right)$ follows from the integration of (3.13) and (1.4) from (3.6) after letting $j \rightarrow+\infty$. Finally, when $\delta=2$, we have

$$
\sup _{[0,1]}\left\|e^{t|x|^{2} / 4\left(t^{2}+R^{2}\right)} u(t)\right\| \leq e^{N\left(1+\|V\|_{\infty}^{2}\right)}\left[\|u(0)\|_{L^{2}\left(\mathbb{R}^{n}\right)}+\left\|e^{|x|^{2} / 4} u(1)\right\|\right],
$$

for all $R>0$. Letting $R \rightarrow 0^{+}$, we get

$$
\sup _{[0,1]}\left\|e^{|x|^{2} / 4 t} u(t)\right\| \leq e^{N\left(1+\|V\|_{\infty}^{2}\right)}\left[\|u(0)\|_{L^{2}\left(\mathbb{R}^{n}\right)}+\left\|e^{|x|^{2} / 4} u(1)\right\|\right],
$$


and it implies, $u \equiv 0$.

Remark 1 . Theorem 1 holds when (1.3) and (1.4) are replaced respectively by

$$
\left\|e^{T x_{1}^{2} / 4\left(T^{2}+R^{2}\right)} u(T)\right\|_{L^{2}\left(\mathbb{R}^{n}\right)}<+\infty
$$

and

$$
\begin{aligned}
& \sup _{[0, T]}\left\|e^{t x_{1}^{2} / 4\left(t^{2}+R^{2}\right)} u(t)\right\|_{L^{2}\left(\mathbb{R}^{n}\right)} \\
& \quad \leq e^{N\left(1+T^{2}\|V\|_{L^{\infty}\left(\mathbb{R}^{n} \times[0, T]\right)}^{2}\right)}\left[\|u(0)\|_{L^{2}\left(\mathbb{R}^{n}\right)}+\left\|e^{T x_{1}^{2} / 4\left(T^{2}+R^{2}\right)} u(T)\right\|_{L^{2}\left(\mathbb{R}^{n}\right)}\right] .
\end{aligned}
$$

\section{REFERENCES}

[1] A. Bonami, B. Demange, A survey on uncertainty principles related to quadratic forms. Collect. Math. Vol. Extra (2006) 1-36.

[2] A. Bonami, B. Demange, P. Jaming, Hermite functions and uncertainty principles for the Fourier and the windowed Fourier transforms. Rev. Mat. Iberoamericana 19,1 (2006) 23-55.

[3] M. Cowling, J. F. Price, Generalizations of Heisenberg's inequality, Harmonic Analysis (Cortona, 1982) Lecture Notes in Math.,992 (1983), 443-449, Springer, Berlin.

[4] M. Cowling, J. F. Price. Bandwidth versus time concentration: the Heisenberg-Pauli-Weyl inequality. SIAM J. Math. Anal. 15 (1984) 151-165.

[5] H. Dong, W. Staubach. Unique continuation for the Schrödinger equation with gradient vector potentials. Proc. Amer. Math. Soc. 135, 7 (2007) 2141-2149.

[6] L. Escauriaza, C.E. Kenig, G. Ponce, L. Vega, On Uniqueness Properties of Solutions of Schrödinger Equations. Comm. PDE. 31, 12 (2006) 1811-1823.

[7] L. Escauriaza, C.E. Kenig, G. Ponce, L. Vega, Decay at Infinity of Caloric Functions within Characteristic Hyperplanes, Math. Res. Letters 13, 3 (2006) 441-453.

[8] L. Escauriaza, C.E. Kenig, G. Ponce, L. Vega, Convexity of Free Solutions of Schrödinger Equations with Gaussian Decay. Math. Res. Lett. 15, 5 (2008) 957-971.

[9] L. Escauriaza, C.E. Kenig, G. Ponce, L. Vega, Hardy's Uncertainty Principle, Convexity and Schrödinger Evolutions. J. Eur. Math. Soc. 10, 4 (2008) 883-907.

[10] L. Escauriaza, C.E. Kenig, G. Ponce, L. Vega. The Sharp Hardy Uncertainty Principle for Schrödinger Evolutions. Duke Math. J. 155, 1 (2010) 163-187.

[11] M. Cowling, L. Escauriaza, C.E. Kenig, G. Ponce, L. Vega. The Hardy Uncertainty Principle Revisited. Indiana U. Math. J. 59, 6 (2010) 2007-2026.

[12] L. Escauriaza, C.E. Kenig, G. Ponce, L. Vega. Uncertainty Principle of Morgan Type for Schrdinger Evolutions. J. London Math. Soc. 83, 1 (2011) 187-207.

[13] L. Escauriaza, C.E. Kenig, G. Ponce, L. Vega. Uniqueness Properties of Solutions to Schrödinger Equations. Bull. (New Series) of the Amer. Math. Soc. 49 (2012) 415-442.

[14] G.H. Hardy, A Theorem Concerning Fourier Transforms, J. London Math. Soc. s1-8 (1933) $227-231$.

[15] L. Hörmander, A uniqueness theorem of Beurling for Fourier transform pairs, Ark. Mat. 29, 2 (1991) 237-240.

[16] A. D. Ionescu, C. E. Kenig, $L^{p}$-Carleman inequalities and uniqueness of solutions of nonlinear Schrödinger equations, Acta Math. 193, 2 (2004) 193-239.

[17] A. D. Ionescu, C. E. Kenig, Uniqueness properties of solutions of Schrödinger equations, J. Funct. Anal. 232 (2006) 90-136.

[18] C.E. Kenig, G. Ponce, L. Vega, On unique continuation for nonlinear Schrödinger equations, Comm. Pure Appl. Math. 60 (2002) 1247-1262.

[19] C.E. Kenig, G. Ponce, L. Vega. A Theorem of Paley-Wiener Type for Schrödinger Evolutions. Annales Scientifiques Ec. Norm. Sup. 47 (2014) 539-557.

[20] A. Sitaram, M. Sundari, S. Thangavelu, Uncertainty principles on certain Lie groups, Proc. Indian Acad. Sci. Math. Sci. 105 (1995), 135-151

[21] E.M. Stein, R. Shakarchi, Princeton Lecture in Analysis II. Complex Analysis, Princeton University Press (2003).

[22] R. Temam, Navier-Stokes Equations, Theory and Numerical Analysis. Amer. Math. Soc (1977). 
(L. Escauriaza) UPV/EHU, Dpto. de Matemáticas, Apto. 644, 48080 Bilbao, Spain.

E-mail address: luis.escauriaza@ehu.eus

(C. E. Kenig) Department of Mathematics, University of Chicago, Chicago, Il. 60637, USA.

E-mail address: cek@math.uchicago.edu

(G. Ponce) Department of Mathematics, University of California, Santa Barbara, CA 93106, USA.

E-mail address: ponce@math.ucsb.edu

(L. Vega) UPV/EHU, Dpto. de Matemáticas, Apto. 644, 48080 Bilbao, Spain.

E-mail address: luis.vega@ehu.eus 